This is the accepted manuscript made available via CHORUS. The article has been published as:

\title{
Observation of the rare decay
}

$B^{\wedge}\{+\} \rightarrow K^{\wedge}\{+\} \Pi^{\wedge}\{0\} \Pi^{\wedge}\{0\}$ and measurement of the quasi-two-body contributions $B^{\wedge}\{+\} \rightarrow K^{*}(892)^{\wedge}\{+\} \pi^{\wedge}\{0\}$, $\mathrm{B}^{\wedge}\{+\} \rightarrow f_{-}\{0\}(980) \mathrm{K}^{\wedge}\{+\}$, and $\mathrm{B}^{\wedge}\{+\} \rightarrow \chi \mathrm{X}_{-}\{\mathrm{C} 0\} \mathrm{K}^{\wedge}\{+\}$

\author{
J. P. Lees et al. (BABAR Collaboration)
}

Phys. Rev. D 84, 092007 - Published 29 November 2011

DOI: 10.1103/PhysRevD.84.092007 
Observation of the rare decay $B^{+} \rightarrow K^{+} \pi^{0} \pi^{0}$ and measurement of the quasi-two-body contributions $B^{+} \rightarrow K^{*}(892)^{+} \pi^{0}, B^{+} \rightarrow f_{0}(980) K^{+}$and $B^{+} \rightarrow \chi_{c 0} K^{+}$

J. P. Lees,${ }^{1}$ V. Poireau, ${ }^{1}$ V. Tisserand,${ }^{1}$ J. Garra Tico, ${ }^{2}$ E. Grauges,${ }^{2}$ M. Martinelli ${ }^{a b},{ }^{3}$ D. A. Milanes ${ }^{a},{ }^{3}$ A. Palano ${ }^{a b},{ }^{3}$ M. Pappagallo ${ }^{a b},{ }^{3}$ G. Eigen,${ }^{4}$ B. Stugu, ${ }^{4}$ D. N. Brown,${ }^{5}$ L. T. Kerth,${ }^{5}$ Yu. G. Kolomensky,${ }^{5}$ G. Lynch, ${ }^{5}$ H. Koch, ${ }^{6}$ T. Schroeder, ${ }^{6}$ D. J. Asgeirsson, ${ }^{7}$ C. Hearty,${ }^{7}$ T. S. Mattison, ${ }^{7}$ J. A. McKenna, ${ }^{7}$ A. Khan, ${ }^{8}$ V. E. Blinov ${ }^{9}$ A. R. Buzykaev,${ }^{9}$ V. P. Druzhinin, ${ }^{9}$ V. B. Golubev,${ }^{9}$ E. A. Kravchenko, ${ }^{9}$ A. P. Onuchin,${ }^{9}$ S. I. Serednyakov ${ }^{9}$ Yu. I. Skovpen, ${ }^{9}$ E. P. Solodov ${ }^{9}$ K. Yu. Todyshev,${ }^{9}$ A. N. Yushkov,${ }^{9}$ M. Bondioli, ${ }^{10}$ D. Kirkby, ${ }^{10}$ A. J. Lankford,${ }^{10}$ M. Mandelkern, ${ }^{10}$ D. P. Stoker, ${ }^{10}$ H. Atmacan, ${ }^{11}$ J. W. Gary, ${ }^{11}$ F. Liu, ${ }^{11}$ O. Long, ${ }^{11}$ G. M. Vitug, ${ }^{11}$ C. Campagnari, ${ }^{12}$ T. M. Hong, ${ }^{12}$ D. Kovalskyi, ${ }^{12}$ J. D. Richman, ${ }^{12}$ C. A. West, ${ }^{12}$ A. M. Eisner,${ }^{13}$ J. Kroseberg, ${ }^{13}$ W. S. Lockman, ${ }^{13}$ A. J. Martinez, ${ }^{13}$ T. Schalk, ${ }^{13}$ B. A. Schumm, ${ }^{13}$ A. Seiden, ${ }^{13}$ C. H. Cheng, ${ }^{14}$ D. A. Doll,,${ }^{14}$ B. Echenard, ${ }^{14}$ K. T. Flood,${ }^{14}$ D. G. Hitlin, ${ }^{14}$ P. Ongmongkolkul,,${ }^{14}$ F. C. Porter, ${ }^{14}$ A. Y. Rakitin,,${ }^{14}$ R. Andreassen, ${ }^{15}$ M. S. Dubrovin, ${ }^{15}$ Z. Huard, ${ }^{15}$ B. T. Meadows,,${ }^{15}$ M. D. Sokoloff,${ }^{15}$ L. Sun, ${ }^{15}$ P. C. Bloom, ${ }^{16}$ W. T. Ford, ${ }^{16}$ A. Gaz, ${ }^{16}$ M. Nagel, ${ }^{16}$ U. Nauenberg, ${ }^{16}$ J. G. Smith, ${ }^{16}$ S. R. Wagner, ${ }^{16}$ R. Ayad $,{ }^{17},{ }^{*}$ W. H. Toki, ${ }^{17}$ B. Spaan, ${ }^{18}$ M. J. Kobel,${ }^{19}$ K. R. Schubert,${ }^{19}$ R. Schwierz, ${ }^{19}$ D. Bernard, ${ }^{20}$ M. Verderi, ${ }^{20}$ P. J. Clark,${ }^{21}$ S. Playfer, ${ }^{21}$ D. Bettoni ${ }^{a},{ }^{22}$ C. Bozzi $^{a},{ }^{22}$ R. Calabrese ${ }^{a b},{ }^{22}$ G. Cibinetto ${ }^{a b},{ }^{22}$ E. Fioravanti ${ }^{a b},{ }^{22}$ I. Garzia $^{a b},{ }^{22}$ E. Luppi $^{a b},{ }^{22}$

M. Munerato ${ }^{a b},{ }^{22}$ M. Negrini ${ }^{a b},{ }^{22}$ L. Piemontese ${ }^{a},{ }^{22}$ V. Santoro, ${ }^{22}$ R. Baldini-Ferroli, ${ }^{23}$ A. Calcaterra,${ }^{23}$

R. de Sangro, ${ }^{23}$ G. Finocchiaro, ${ }^{23}$ M. Nicolaci, ${ }^{23}$ P. Patteri,${ }^{23}$ I. M. Peruzzi,${ }^{23, \dagger}$ M. Piccolo, ${ }^{23}$ M. Rama, ${ }^{23}$ A. Zallo, ${ }^{23}$ R. Contri ${ }^{a b},{ }^{24}$ E. Guido ${ }^{a b},{ }^{24}$ M. Lo Vetere ${ }^{a b},{ }^{24}$ M. R. Monge ${ }^{a b},{ }^{24}$ S. Passaggio ${ }^{a},{ }^{24}$ C. Patrignani ${ }^{a b},{ }^{24}$ E. Robutti ${ }^{a},{ }^{24}$ B. Bhuyan, ${ }^{25}$ V. Prasad,${ }^{25}$ C. L. Lee, ${ }^{26}$ M. Morii, ${ }^{26}$ A. J. Edwards, ${ }^{27}$ A. Adametz, ${ }^{28}$ J. Marks, ${ }^{28}$

U. Uwer, ${ }^{28}$ F. U. Bernlochner ${ }^{29}$ M. Ebert ${ }^{29}$ H. M. Lacker, ${ }^{29}$ T. Lueck, ${ }^{29}$ P. D. Dauncey,${ }^{30}$ M. Tibbetts ${ }^{30}$ P. K. Behera, ${ }^{31}$ U. Mallik, ${ }^{31}$ C. Chen, ${ }^{32}$ J. Cochran, ${ }^{32}$ W. T. Meyer, ${ }^{32}$ S. Prell, ${ }^{32}$ E. I. Rosenberg, ${ }^{32}$ A. E. Rubin, ${ }^{32}$ A. V. Gritsan, ${ }^{33}$ Z. J. Guo, ${ }^{33}$ N. Arnaud ${ }^{34}$ M. Davier, ${ }^{34}$ G. Grosdidier, ${ }^{34}$ F. Le Diberder,${ }^{34}$ A. M. Lutz, ${ }^{34}$ B. Malaescu, ${ }^{34}$ P. Roudeau,${ }^{34}$ M. H. Schune, ${ }^{34}$ A. Stocchi, ${ }^{34}$ G. Wormser,${ }^{34}$ D. J. Lange,${ }^{35}$ D. M. Wright,${ }^{35}$ I. Bingham ${ }^{36}$ C. A. Chavez, ${ }^{36}$ J. P. Coleman, ${ }^{36}$ J. R. Fry ${ }^{36}$ E. Gabathuler, ${ }^{36}$ D. E. Hutchcroft, ${ }^{36}$ D. J. Payne ${ }^{36}$ C. Touramanis, ${ }^{36}$ A. J. Bevan, ${ }^{37}$ F. Di Lodovico, ${ }^{37}$ R. Sacco,${ }^{37}$ M. Sigamani, ${ }^{37}$ G. Cowan, ${ }^{38}$ D. N. Brown, ${ }^{39}$ C. L. Davis, ${ }^{39}$ A. G. Denig, ${ }^{40}$ M. Fritsch,${ }^{40}$ W. Gradl, ${ }^{40}$ A. Hafner, ${ }^{40}$ E. Prencipe,${ }^{40}$ K. E. Alwyn, ${ }^{41}$ D. Bailey, ${ }^{41}$

R. J. Barlow ${ }^{41,}{ }^{\ddagger}$ G. Jackson, ${ }^{41}$ G. D. Lafferty, ${ }^{41}$ E. Behn,${ }^{42}$ R. Cenci,${ }^{42}$ B. Hamilton, ${ }^{42}$ A. Jawahery ${ }^{42}$

D. A. Roberts, ${ }^{42}$ G. Simi,${ }^{42}$ C. Dallapiccola ${ }^{43}$ R. Cowan, ${ }^{44}$ D. Dujmic, ${ }^{44}$ G. Sciolla, ${ }^{44}$ D. Lindemann, ${ }^{45}$ P. M. Patel, ${ }^{45}$ S. H. Robertson, ${ }^{45}$ M. Schram,${ }^{45}$ P. Biassoni ${ }^{a b},{ }^{46}$ A. Lazzaro ${ }^{a b}{ }^{46}$ V. Lombardo ${ }^{a},{ }^{46}$ N. Neri ${ }^{a b},{ }^{46}$ F. Palombo ${ }^{a b}{ }^{46}$ S. Stracka ${ }^{a b},{ }^{46}$ L. Cremaldi, ${ }^{47}$ R. Godang, ${ }^{47}, \S$ R. Kroeger, ${ }^{47}$ P. Sonnek, ${ }^{47}$ D. J. Summers, ${ }^{47}$ X. Nguyen, ${ }^{48}$ P. Taras ${ }^{48}$ G. De Nardo ${ }^{a b},{ }^{49}$ D. Monorchio ${ }^{a b},{ }^{49}$ G. Onorato ${ }^{a b},{ }^{49}$ C. Sciacca ${ }^{a b},{ }^{49}$ G. Raven, ${ }^{50}$ H. L. Snoek, ${ }^{50}$ C. P. Jessop, ${ }^{51}$ K. J. Knoepfel ${ }^{51}$ J. M. LoSecco, ${ }^{51}$ W. F. Wang, ${ }^{51}$ K. Honscheid, ${ }^{52}$ R. Kass, ${ }^{52}$ J. Brau, ${ }^{53}$ R. Frey ${ }^{53}$ N. B. Sinev ${ }^{53}$ D. Strom, ${ }^{53}$ E. Torrence, ${ }^{53}$ E. Feltresi ${ }^{a b},{ }^{54}$ N. Gagliardi ${ }^{a b},{ }^{54}$ M. Margoni ${ }^{a b},{ }^{54}$ M. Morandin ${ }^{a},{ }^{54}$ M. Posocco ${ }^{a},{ }^{54}$ M. Rotondo ${ }^{a}{ }^{54}$ F. Simonetto ${ }^{a b},{ }^{54}$ R. Stroili ${ }^{a b},{ }^{54}$ S. Akar, ${ }^{55}$ E. Ben-Haim, ${ }^{55}$ M. Bomben, ${ }^{55}$ G. R. Bonneaud, ${ }^{55}$ H. Briand,${ }^{55}$ G. Calderini, ${ }^{55}$ J. Chauveau, ${ }^{55}$ O. Hamon, ${ }^{55}$ Ph. Leruste ${ }^{55}$ G. Marchiori, ${ }^{55}$ J. Ocariz, ${ }^{55}$ S. Sitt, ${ }^{55}$ M. Biasini ${ }^{a b},{ }^{56}$ E. Manoni ${ }^{a b},{ }^{56}$ S. Pacetti ${ }^{a b},{ }^{56}$ A. Rossi ${ }^{a b},{ }^{56}$ C. Angelini ${ }^{a b},{ }^{57}$ G. Batignani ${ }^{a b}, 57$ S. Bettarini ${ }^{a b}, 57$ M. Carpinelli ${ }^{a b}, 57$, G. Casarosa ${ }^{a b},{ }^{57}$ A. Cervelli ${ }^{a b}, 57$ F. Forti ${ }^{a b}, 57$

M. A. Giorgi ${ }^{a b}, 57$ A. Lusiani ${ }^{a c},{ }^{57}$ B. Oberhof ${ }^{a b},{ }^{57}$ E. Paoloni ${ }^{a b},{ }^{57}$ A. Perez ${ }^{a},{ }^{57}$ G. Rizzo ${ }^{a b},{ }^{57}$ J. J. Walsh ${ }^{a}, 57$ D. Lopes Pegna ${ }^{58}$ C. Lu, ${ }^{58}$ J. Olsen,${ }^{58}$ A. J. S. Smith, ${ }^{58}$ A. V. Telnov ${ }^{58}$ F. Anulli ${ }^{a}{ }^{59}$ G. Cavoto ${ }^{a},{ }^{59}$ R. Faccini ${ }^{a b},{ }^{59}$

F. Ferrarotto ${ }^{a},{ }^{59}$ F. Ferroni ${ }^{a b},{ }^{59}$ M. Gaspero ${ }^{a b},{ }^{59}$ L. Li Gioi ${ }^{a},{ }^{59}$ M. A. Mazzoni ${ }^{a},{ }^{59}$ G. Piredda ${ }^{a},{ }^{59}$ C. Bünger ${ }^{60}$ O. Grünberg, ${ }^{60}$ T. Hartmann, ${ }^{60}$ T. Leddig, ${ }^{60}$ H. Schröder, ${ }^{60}$ R. Waldi,${ }^{60}$ T. Adye,${ }^{61}$ E. O. Olaiya, ${ }^{61}$ F. F. Wilson, ${ }^{61}$ S. Emery ${ }^{62}$ G. Hamel de Monchenault,${ }^{62}$ G. Vasseur, ${ }^{62}$ Ch. Yèche,${ }^{62}$ D. Aston, ${ }^{63}$ D. J. Bard, ${ }^{63}$ R. Bartoldus, ${ }^{63}$ C. Cartaro, ${ }^{63}$ M. R. Convery ${ }^{63}$ J. Dorfan ${ }^{63}$ G. P. Dubois-Felsmann ${ }^{63}$ W. Dunwoodie, ${ }^{63}$ R. C. Field, ${ }^{63}$ M. Franco Sevilla, ${ }^{63}$ B. G. Fulsom, ${ }^{63}$ A. M. Gabareen, ${ }^{63}$ M. T. Graham, ${ }^{63}$ P. Grenier, ${ }^{63}$ C. Hast,${ }^{63}$ W. R. Innes, ${ }^{63}$ M. H. Kelsey, ${ }^{63}$ H. Kim, ${ }^{63}$ P. Kim, ${ }^{63}$ M. L. Kocian, ${ }^{63}$ D. W. G. S. Leith ${ }^{63}$ P. Lewis, ${ }^{63}$ S. Li, ${ }^{63}$ B. Lindquist ${ }^{63}$ S. Luitz,${ }^{63}$ V. Luth,${ }^{63}$ H. L. Lynch, ${ }^{63}$ D. B. MacFarlane, ${ }^{63}$ D. R. Muller, ${ }^{63}$ H. Neal,${ }^{63}$ S. Nelson,${ }^{63}$ I. Ofte, ${ }^{63}$ M. Perl,${ }^{63}$ T. Pulliam, ${ }^{63}$ B. N. Ratcliff, ${ }^{63}$ A. Roodman, ${ }^{63}$ A. A. Salnikov, ${ }^{63}$ R. H. Schindler ${ }^{63}$ A. Snyder, ${ }^{63}$ D. Su, ${ }^{63}$ M. K. Sullivan, ${ }^{63}$ J. Va'vra, ${ }^{63}$ A. P. Wagner ${ }^{63}$ M. Weaver, ${ }^{63}$ W. J. Wisniewski, ${ }^{63}$ M. Wittgen, ${ }^{63}$ D. H. Wright, ${ }^{63}$

H. W. Wulsin, ${ }^{63}$ A. K. Yarritu, ${ }^{63}$ C. C. Young, ${ }^{63}$ V. Ziegler ${ }^{63}$ W. Park, ${ }^{64}$ M. V. Purohit,${ }^{64}$ R. M. White ${ }^{64}$ J. R. Wilson, ${ }^{64}$ A. Randle-Conde, ${ }^{65}$ S. J. Sekula, ${ }^{65}$ M. Bellis, ${ }^{66}$ J. F. Benitez, ${ }^{66}$ P. R. Burchat, ${ }^{66}$ T. S. Miyashita, ${ }^{66}$ M. S. Alam, ${ }^{67}$ J. A. Ernst, ${ }^{67}$ R. Gorodeisky, ${ }^{68}$ N. Guttman, ${ }^{68}$ D. R. Peimer, ${ }^{68}$ A. Soffer, ${ }^{68}$ P. Lund, ${ }^{69}$ S. M. Spanier, ${ }^{69}$ R. Eckmann, ${ }^{70}$ J. L. Ritchie, ${ }^{70}$ A. M. Ruland,${ }^{70}$ C. J. Schilling, ${ }^{70}$ R. F. Schwitters, ${ }^{70}$ B. C. Wray,${ }^{70}$ 
J. M. Izen, ${ }^{71}$ X. C. Lou, ${ }^{71}$ F. Bianchi ${ }^{a b},{ }^{72}$ D. Gamba ${ }^{a b},{ }^{72}$ L. Lanceri ${ }^{a b},{ }^{73}$ L. Vitale ${ }^{a b},{ }^{73}$ F. Martinez-Vidal, ${ }^{74}$ A. Oyanguren, ${ }^{74}$ H. Ahmed, ${ }^{75}$ J. Albert, ${ }^{75}$ Sw. Banerjee, ${ }^{75}$ H. H. F. Choi, ${ }^{75}$ G. J. King, ${ }^{75}$ R. Kowalewski, ${ }^{75}$ M. J. Lewczuk, ${ }^{75}$ I. M. Nugent, ${ }^{75}$ J. M. Roney, ${ }^{75}$ R. J. Sobie, ${ }^{75}$ N. Tasneem, ${ }^{75}$ T. J. Gershon, ${ }^{76}$ P. F. Harrison, ${ }^{76}$ T. E. Latham, ${ }^{76}$ E. M. T. Puccio, ${ }^{76}$ H. R. Band, ${ }^{77}$ S. Dasu, ${ }^{77}$ Y. Pan, ${ }^{77}$ R. Prepost,${ }^{77}$ and S. L. Wu ${ }^{77}$

(The BABAR Collaboration)

\footnotetext{
${ }^{1}$ Laboratoire d'Annecy-le-Vieux de Physique des Particules (LAPP),
}

Université de Savoie, CNRS/IN2P3, F-74941 Annecy-Le-Vieux, France

${ }^{2}$ Universitat de Barcelona, Facultat de Fisica, Departament ECM, E-08028 Barcelona, Spain

${ }^{3}$ INFN Sezione di Bari ${ }^{a}$; Dipartimento di Fisica, Università di Bari ${ }^{b}$, I-70126 Bari, Italy

${ }^{4}$ University of Bergen, Institute of Physics, N-5007 Bergen, Norway

${ }^{5}$ Lawrence Berkeley National Laboratory and University of California, Berkeley, California 94720, USA

${ }^{6}$ Ruhr Universität Bochum, Institut für Experimentalphysik 1, D-44780 Bochum, Germany

${ }^{7}$ University of British Columbia, Vancouver, British Columbia, Canada V6T $1 Z 1$

${ }^{8}$ Brunel University, Uxbridge, Middlesex UB8 3PH, United Kingdom

${ }^{9}$ Budker Institute of Nuclear Physics, Novosibirsk 630090, Russia

${ }^{10}$ University of California at Irvine, Irvine, California 92697, USA

${ }^{11}$ University of California at Riverside, Riverside, California 92521, USA

${ }^{12}$ University of California at Santa Barbara, Santa Barbara, California 93106, USA

${ }^{13}$ University of California at Santa Cruz, Institute for Particle Physics, Santa Cruz, California 95064, USA

${ }^{14}$ California Institute of Technology, Pasadena, California 91125, USA

${ }^{15}$ University of Cincinnati, Cincinnati, Ohio 45221, USA

${ }^{16}$ University of Colorado, Boulder, Colorado 80309, USA

${ }^{17}$ Colorado State University, Fort Collins, Colorado 80523, USA

${ }^{18}$ Technische Universität Dortmund, Fakultät Physik, D-44221 Dortmund, Germany

${ }^{19}$ Technische Universität Dresden, Institut für Kern- und Teilchenphysik, D-01062 Dresden, Germany

${ }^{20}$ Laboratoire Leprince-Ringuet, Ecole Polytechnique, CNRS/IN2P3, F-91128 Palaiseau, France

${ }^{21}$ University of Edinburgh, Edinburgh EH9 3JZ, United Kingdom

${ }^{22}$ INFN Sezione di Ferrara $^{a}$; Dipartimento di Fisica, Università di Ferrara ${ }^{b}$, I-44100 Ferrara, Italy

${ }^{23}$ INFN Laboratori Nazionali di Frascati, I-00044 Frascati, Italy

${ }^{24}$ INFN Sezione di Genova $^{a}$; Dipartimento di Fisica, Università di Genova ${ }^{b}$, I-16146 Genova, Italy

${ }^{25}$ Indian Institute of Technology Guwahati, Guwahati, Assam, 781 039, India

${ }^{26}$ Harvard University, Cambridge, Massachusetts 02138, USA

${ }^{27}$ Harvey Mudd College, Claremont, California 91711

${ }^{28}$ Universität Heidelberg, Physikalisches Institut, Philosophenweg 12, D-69120 Heidelberg, Germany

${ }^{29}$ Humboldt-Universität zu Berlin, Institut für Physik, Newtonstr. 15, D-12489 Berlin, Germany

${ }^{30}$ Imperial College London, London, SW7 2AZ, United Kingdom

${ }^{31}$ University of Iowa, Iowa City, Iowa 52242, USA

${ }^{32}$ Iowa State University, Ames, Iowa 50011-3160, USA

${ }^{33}$ Johns Hopkins University, Baltimore, Maryland 21218, USA

${ }^{34}$ Laboratoire de l'Accélérateur Linéaire, IN2P3/CNRS et Université Paris-Sud 11,

Centre Scientifique d'Orsay, B. P. 34, F-91898 Orsay Cedex, France

${ }^{35}$ Lawrence Livermore National Laboratory, Livermore, California 94550, USA

${ }^{36}$ University of Liverpool, Liverpool L69 7ZE, United Kingdom

${ }^{37}$ Queen Mary, University of London, London, E1 4NS, United Kingdom

${ }^{38}$ University of London, Royal Holloway and Bedford New College, Egham, Surrey TW20 0EX, United Kingdom

${ }^{39}$ University of Louisville, Louisville, Kentucky 40292, USA

${ }^{40}$ Johannes Gutenberg-Universität Mainz, Institut für Kernphysik, D-55099 Mainz, Germany

${ }^{41}$ University of Manchester, Manchester M13 9PL, United Kingdom

${ }^{42}$ University of Maryland, College Park, Maryland 20742, USA

${ }^{43}$ University of Massachusetts, Amherst, Massachusetts 01003, USA

${ }^{44}$ Massachusetts Institute of Technology, Laboratory for Nuclear Science, Cambridge, Massachusetts 02139, USA

${ }^{45}$ McGill University, Montréal, Québec, Canada H3A $2 T 8$

${ }^{46}$ INFN Sezione di Milano $^{a}$; Dipartimento di Fisica, Università di Milano ${ }^{b}$, I-20133 Milano, Italy

${ }^{47}$ University of Mississippi, University, Mississippi 38677, USA

${ }^{48}$ Université de Montréal, Physique des Particules, Montréal, Québec, Canada H3C $3 J^{7}$

${ }^{49}$ INFN Sezione di Napoli ${ }^{a}$; Dipartimento di Scienze Fisiche,

Università di Napoli Federico $I^{b}$, I-80126 Napoli, Italy

${ }^{50}$ NIKHEF, National Institute for Nuclear Physics and High Energy Physics, NL-1009 DB Amsterdam, The Netherlands

${ }^{51}$ University of Notre Dame, Notre Dame, Indiana 46556, USA

${ }^{52}$ Ohio State University, Columbus, Ohio 43210, USA

${ }^{53}$ University of Oregon, Eugene, Oregon 97403, USA

${ }^{54}$ INFN Sezione di Padova $^{a}$; Dipartimento di Fisica, Università di Padova ${ }^{b}$ I-35131 Padova, Italy 




We report an analysis of charmless hadronic decays of charged $B$ mesons to the final state $K^{+} \pi^{0} \pi^{0}$, using a data sample of $470.9 \pm 2.8$ million $B \bar{B}$ events collected with the BABAR detector at the $\Upsilon(4 S)$ resonance. We observe an excess of signal events, with a significance above 10 standard deviations including systematic uncertainties, and measure the branching fraction and $C P$ asymmetry to be $\mathcal{B}\left(B^{+} \rightarrow K^{+} \pi^{0} \pi^{0}\right)=(16.2 \pm 1.2 \pm 1.5) \times 10^{-6}$ and $A_{C P}\left(B^{+} \rightarrow K^{+} \pi^{0} \pi^{0}\right)=-0.06 \pm 0.06 \pm 0.04$, where the uncertainties are statistical and systematic, respectively. Additionally, we study the contributions of the $B^{+} \rightarrow K^{*}(892)^{+} \pi^{0}, B^{+} \rightarrow f_{0}(980) K^{+}$, and $B^{+} \rightarrow \chi_{c 0} K^{+}$quasi-two-body decays. We report the world's best measurements of the branching fraction and $C P$ asymmetry of the $B^{+} \rightarrow K^{+} \pi^{0} \pi^{0}$ and $B^{+} \rightarrow K^{*}(892)^{+} \pi^{0}$ channels.

PACS numbers: 13.25.Hw, 11.30.Er, 12.39.-x

\section{INTRODUCTION}

Recent measurements of rates and asymmetries in $B \rightarrow K \pi$ decays have generated considerable interest because of possible hints of new physics contributions $[1,2]$. Unfortunately, hadronic uncertainties prevent a clear interpretation of these results in terms of physics beyond the Standard Model (SM). A data-driven approach involving measurements of all observables in the $B \rightarrow K \pi$ system can in principle resolve the theoretical situation,

\footnotetext{
*Now at Temple University, Philadelphia, Pennsylvania 19122, USA

${ }^{\dagger}$ Also with Università di Perugia, Dipartimento di Fisica, Perugia, Italy

$¥$ Now at the University of Huddersfield, Huddersfield HD1 3DH, UK

$\S$ Now at University of South Alabama, Mobile, Alabama 36688, USA

đAlso with Università di Sassari, Sassari, Italy
}

but much more precise measurements are needed [3-5].

The ratios of tree-to-penguin amplitudes in the related pseudoscalar-vector decays $B \rightarrow K^{*} \pi$ and $B \rightarrow K \rho$ are predicted to be two to three times larger than those in $B \rightarrow K \pi$. Hence, these decays could have considerably larger $C P$ asymmetries and thus provide useful additional information [6-8]. In Table I we review the existing experimental measurements of the channels in the $B \rightarrow K^{*} \pi$ system. Improved measurements of the $K^{*+} \pi^{0}$ [9] decay can be obtained using the full $\Upsilon(4 S)$ $B A B A R$ dataset.

TABLE I: Experimental measurements of $B \rightarrow K^{*} \pi$ decays. Average values come from HFAG [10].

\begin{tabular}{cccc}
\hline Mode & $\mathcal{B} \times 10^{6}$ & $A_{C P}$ & References \\
\hline$K^{*+} \pi^{-}$ & $10.3 \pm 1.1$ & $-0.23 \pm 0.08$ & {$[11-14]$} \\
$K^{*+} \pi^{0}$ & $6.9 \pm 2.3$ & $0.04 \pm 0.29 \pm 0.05$ & {$[15]$} \\
$K^{* 0} \pi^{+}$ & $9.9_{-0.9}^{+0.8}$ & $-0.020_{-0.061}^{+0.067}$ & {$[16,17]$} \\
$K^{* 0} \pi^{0}$ & $2.4 \pm 0.7$ & $-0.15 \pm 0.12 \pm 0.02$ & {$[12,18]$} \\
\hline
\end{tabular}

The four $K^{*} \pi$ decays populate six $K \pi \pi$ Dalitz plots 
(the four $K \rho$ decays also produce four of the same six final states). To date, Dalitz plot analyses have been performed in the channels $K^{+} \pi^{+} \pi^{-}[16,17], K_{S}^{0} \pi^{+} \pi^{-}[14$, 19] and $K^{+} \pi^{-} \pi^{0}[12,20]$. The first two of these have shown the presence of a poorly-understood structure, dubbed the $f_{\mathrm{X}}(1300)$, in the $\pi^{+} \pi^{-}$invariant mass distribution. A study of the invariant mass spectrum in $B^{+} \rightarrow K^{+} \pi^{0} \pi^{0}$ decays could help elucidate the nature of this peak, since even-spin states will populate both $K \pi^{+} \pi^{-}$and $K \pi^{0} \pi^{0}$ (assuming isospin symmetry), while odd-spin states cannot decay to $\pi^{0} \pi^{0}$.

Knowledge of the dominant contributions to the $K^{+} \pi^{0} \pi^{0}$ Dalitz plot may also help to clarify the interpretation of the inclusive time-dependent analyses [21] of $B^{0} \rightarrow K_{S}^{0} \pi^{0} \pi^{0}$ [22]. For such $b \rightarrow s$ penguin-dominated decays the naïve Standard Model expectation is that the time-dependent $C P$ violation parameter should be given by $S_{C P} \approx-\eta_{C P} \sin (2 \beta)$, where $\eta_{C P}$ is the $C P$ eigenvalue of the final state $\left(+1\right.$ for $\left.K_{S}^{0} \pi^{0} \pi^{0}\right)$ and $\beta$ is an angle of the Cabibbo-Kobayashi-Maskawa [23, 24] unitarity triangle. Currently, the results for $B^{0} \rightarrow K_{S}^{0} \pi^{0} \pi^{0}$ show the largest deviation, among hadronic $b \rightarrow s$ penguin-dominated decays [10], from the angle $\beta$ measured in charmed decays, albeit with a large uncertainty. Such deviations could be caused by new physics, but in order to rule out the possibility of sizeable corrections to the Standard Model prediction, better understanding of the population of the $K \pi^{0} \pi^{0}$ Dalitz plots is necessary.

In this article, we present the results of a search for the three-body decay $B^{+} \rightarrow K^{+} \pi^{0} \pi^{0}$, including shortlived intermediate two-body modes that decay to this final state. A full amplitude analysis of the three-body decay would require detailed understanding of effects related to the misreconstruction of signal events, such as the smearing of their Dalitz plot positions. These effects are significant in the final state under study, which involves two neutral pions. Therefore, in order to avoid heavy reliance on Monte Carlo (MC) simulations, we do not perform a Dalitz plot analysis, but instead extract information on intermediate modes including narrow resonances $\left(K^{*+}(892) \pi^{0}, f_{0}(980) K^{+}\right.$and $\left.\chi_{c 0} K^{+}\right)$by studying the two-body invariant mass distributions.

There is no existing previous measurement of the three-body branching fraction, but several quasi-twobody modes that can decay to this final state have been seen, with varying significances. These include $B^{+} \rightarrow f_{0}(980) K^{+}$, observed in the $f_{0}(980) \rightarrow \pi^{+} \pi^{-}$ channel $[16,17]$ and also seen in $f_{0}(980) \rightarrow K^{+} K^{-}[25]$; $B^{+} \rightarrow f_{2}(1270) K^{+}$, seen in $f_{2}(1270) \rightarrow \pi^{+} \pi^{-}[16,17]$; and $B^{+} \rightarrow K^{*+}(892) \pi^{0}$, seen in $K^{*+}(892) \rightarrow K^{+} \pi^{0}$ [15]. The decay $B^{+} \rightarrow \chi_{c 0} K^{+}$has also been observed with $\chi_{c 0} \rightarrow \pi^{+} \pi^{-}[16,17]$ and $\chi_{c 0} \rightarrow K^{+} K^{-}[25,26]$.

\section{EVENT RECONSTRUCTION AND SELECTION}

The data used in the analysis were collected with the $B A B A R$ detector [27] at the PEP-II asymmetric-energy $e^{+} e^{-}$collider at the SLAC National Accelerator Laboratory. The sample consists of an integrated luminosity of $429 \mathrm{fb}^{-1}$ recorded at the $\Upsilon(4 S)$ resonance ("onpeak") and $45 \mathrm{fb}^{-1}$ collected $40 \mathrm{MeV}$ below the resonance ("off-peak"). The on-peak data sample contains the full BABAR $\Upsilon(4 S)$ dataset, consisting of $470.9 \pm 2.8$ million $B \bar{B}$ events.

We reconstruct $B^{+} \rightarrow K^{+} \pi^{0} \pi^{0}$ decay candidates by combining a $K^{+}$candidate with two neutral pion candidates. The $K^{+}$candidate is a charged track with transverse momentum above $0.05 \mathrm{GeV} / c$ that is consistent with having originated at the interaction region. Separation of charged kaons from charged pions is accomplished with energy-loss information from the tracking subdetectors and with the Cherenkov angle and number of photons measured by a ring-imaging Cherenkov detector. The efficiency for kaon selection is approximately $80 \%$ including geometrical acceptance, while the probability of misidentification of pions as kaons is below $5 \%$ up to a laboratory momentum of $4 \mathrm{GeV} / c$. Neutral pion candidates are formed from pairs of neutral clusters with laboratory energies above $0.05 \mathrm{GeV}$ and lateral moments [28] between 0.01 and 0.6. We require the mass of the reconstructed $\pi^{0}$ to be within the range $0.115 \mathrm{GeV} / c^{2}<m_{\gamma \gamma}<0.150 \mathrm{GeV} / c^{2}$ and the absolute value of the cosine of the decay angle in the $\pi^{0}$ rest frame to be less than 0.9. Figure 1 shows the distribution of the mass of neutral pion candidates in on-peak data. Following this selection, when forming the $B$ candidate, the $\pi^{0}$ candidates have their masses constrained to the world average value [29].

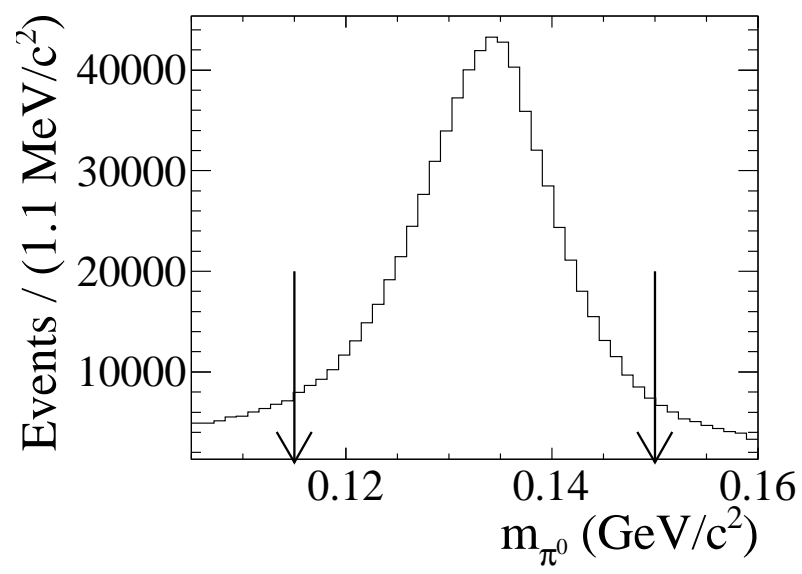

FIG. 1: Masses of $\pi^{0}$ candidates in on-peak data. The arrows indicate the selection requirements.

We exclude candidates consistent with the $B^{+} \rightarrow$ $K_{S}^{0} K^{+}, K_{S}^{0} \rightarrow \pi^{0} \pi^{0}$ decay chain by rejecting events with 
a pair of $\pi^{0}$ mesons that satisfies $0.40 \mathrm{GeV} / c^{2}<m_{\pi^{0} \pi^{0}}<$ $0.55 \mathrm{GeV} / c^{2}$. This veto has a signal efficiency of at least $96 \%$ for any charmless resonant decay and of almost $100 \%$ for nonresonant $B^{+} \rightarrow K^{+} \pi^{0} \pi^{0}$ and $B^{+} \rightarrow \chi_{c 0} K^{+}$ decays.

Because of the presence of two neutral pions in the final state, there is a significant probability for signal events to be misreconstructed, due to low momentum photons that are replaced by photons from the decay of the other $B$ meson in the event. We refer to these as "self-crossfeed" (SCF) events, as opposed to correctly reconstructed (CR) events. Using a classification based on Monte Carlo information, we find that in simulated events the SCF fraction depends strongly on the resonant substructure of the signal, and ranges from $2 \%$ for $B^{+} \rightarrow \chi_{c 0} K^{+}$ decays to $30 \%$ for $B^{+} \rightarrow f_{2}(1270) K^{+}$decays.

In order to suppress the contribution arising from the dominant background, due to continuum $e^{+} e^{-} \rightarrow$ $q \bar{q}(q=u, d, s, c)$ events, we employ a neural network that combines four variables commonly used to discriminate jet-like $q \bar{q}$ events from the more spherical $B \bar{B}$ events. The first of these is the ratio of the second-to-zeroth order momentum-weighted Legendre polynomial moments,

$$
\frac{L_{2}}{L_{0}}=\frac{\sum_{i \in \mathrm{ROE}} \frac{1}{2}\left(3 \cos ^{2} \theta_{i}-1\right) p_{i}}{\sum_{i \in \mathrm{ROE}} p_{i}},
$$

where the summations are over all tracks and neutral clusters in the event excluding those that form the $B$ candidate (the "rest of the event" or ROE), $p_{i}$ is the particle momentum and $\theta_{i}$ is the angle between the particle and the thrust axis of the $B$ candidate. The three other variables entering the neural network are the absolute value of the cosine of the angle between the $B$ direction and the beam axis, the absolute value of the cosine of the angle between the $B$ thrust axis and the beam axis, and the absolute value of the output of a neural network used for "flavor tagging", i.e. , for distinguishing $B$ from $\bar{B}$ decays using inclusive properties of the decay of the other $B$ meson in the $\Upsilon(4 S) \rightarrow B \bar{B}$ event [30]. The first three quantities are calculated in the center-of-mass $(\mathrm{CM})$ frame. The neural network is trained on a sample of signal MC and off-peak data. We apply a loose criterion on the neural network output $\left(\mathrm{NN}_{\text {out }}\right)$, which retains approximately $90 \%$ of the signal while rejecting approximately $82 \%$ of the $q \bar{q}$ background.

In addition to $\mathrm{NN}_{\text {out }}$, we distinguish signal from background events using two kinematic variables:

$$
\begin{aligned}
m_{\mathrm{ES}} & =\sqrt{E_{\mathrm{X}}^{2}-\mathbf{p}_{B}^{2}}, \\
\Delta E & =E_{B}^{\star}-\sqrt{s} / 2,
\end{aligned}
$$

where

$$
E_{\mathrm{X}}=\left(s / 2+\mathbf{p}_{i} \cdot \mathbf{p}_{B}\right) / E_{i},
$$

$\sqrt{s}$ is the total CM energy, $\left(E_{i}, \mathbf{p}_{i}\right)$ and $\left(E_{B}, \mathbf{p}_{B}\right)$ are the four-momenta of the initial $e^{+} e^{-}$system and $B$ candidate, respectively, both measured in the lab frame, while the star indicates the $\mathrm{CM}$ frame. The signal $m_{\mathrm{ES}}$ distribution for CR events is approximately independent of the $B^{+} \rightarrow K^{+} \pi^{0} \pi^{0}$ Dalitz plot distribution and peaks near the $B$ mass with a resolution of about $3 \mathrm{MeV} / c^{2}$. We select signal candidates with $5.260 \mathrm{GeV} / c^{2}<m_{\mathrm{ES}}<$ $5.286 \mathrm{GeV} / c^{2}$. The $\mathrm{CR}$ signal $\Delta E$ distribution peaks near zero, but has a resolution that depends on the event-by-event Dalitz plot position, the PDF of which is a priori unknown. Prior to the selection of multiple candidates (see below), we make the requirement $|\Delta E|<0.30 \mathrm{GeV}$, in order to retain sidebands for background studies. However, to avoid possible biases [31] we do not use $\Delta E$ in the fit described below and instead apply tighter selection criteria for events entering the fit, $-0.15 \mathrm{GeV}<\Delta E<0.05 \mathrm{GeV}$. These criteria have an efficiency of about $80 \%$ for signal while retaining only about $30 \%$ of the background, both compared to the looser requirement $|\Delta E|<0.30 \mathrm{GeV}$.

The efficiency for signal events to pass all the selection criteria is determined as a function of position in the Dalitz plot. Using an MC simulation in which events uniformly populate phase space, we obtain an average efficiency of approximately $16 \%$, though values as low as $8 \%$ are found near the corners of the Dalitz plot, where one of the particles is soft.

An average of $1.3 \mathrm{~B}$ candidates is found per selected event. In events with multiple candidates we choose the one with the smallest value of a $\chi^{2}$ variable formed from the sum of the $\chi^{2}$ values of the two $\pi^{0}$ candidate masses, calculated from the difference between the reconstructed $\pi^{0}$ mass with respect to the nominal $\pi^{0}$ mass. This procedure has been found to select the best reconstructed candidate more than $90 \%$ of the time, and does not bias our fit variables.

We study residual background contributions from $B \bar{B}$ events using $\mathrm{MC}$ simulations. We divide these events into four categories based on their shapes in the $m_{\mathrm{ES}}$ and $\Delta E$ distributions. The first category comprises twobody modes (mainly $B^{+} \rightarrow K^{+} \pi^{0}$ ); the second contains three-body modes (mainly $B^{+} \rightarrow K^{*+}\left(\rightarrow K^{+} \pi^{0}\right) \gamma$ and $\left.B^{+} \rightarrow \pi^{+} \pi^{0} \pi^{0}\right)$; the third and fourth are composed of higher multiplicity decays (many possible sources with or without intermediate charmed states) with missing particles, and are distinguished by the absence or presence of a peak in the $m_{\mathrm{ES}}$ distribution, respectively. Based on the MC-derived efficiencies, total number of $B \bar{B}$ events, and known branching fractions $[10,29]$, we expect $70 \pm 9$, $39 \pm 18,1090 \pm 40$ and $170 \pm 30$ events in the four categories respectively.

\section{STUDY OF THE INCLUSIVE $B^{+} \rightarrow K^{+} \pi^{0} \pi^{0}$ DECAY}

To obtain the $B^{+} \rightarrow K^{+} \pi^{0} \pi^{0}$ signal yield, we perform an unbinned extended maximum likelihood fit to the candidate events using two input variables: $m_{\mathrm{ES}}$ and $\mathrm{NN}_{\text {out }}$. For each component $j$ (signal, $q \bar{q}$ background, and the 
four $B \bar{B}$ background categories), we define a probability density function (PDF)

$$
\mathcal{P}_{j}^{i} \equiv \mathcal{P}_{j}\left(m_{\mathrm{ES}}{ }^{i}\right) \mathcal{P}_{j}\left(\mathrm{NN}_{\text {out }}{ }^{i}\right),
$$

where the index $i$ runs over the selected events. The signal component is further separated into CR and SCF parts

$$
\begin{array}{r}
\mathcal{P}_{\mathrm{sig}}^{i} \equiv\left(1-f_{\mathrm{SCF}}\right) \mathcal{P}_{\mathrm{CR}}\left(m_{\mathrm{ES}}{ }^{i}\right) \mathcal{P}_{\mathrm{CR}}\left(\mathrm{NN}_{\text {out }}{ }^{i}\right)+ \\
f_{\mathrm{SCF}} \mathcal{P}_{\mathrm{SCF}}\left(m_{\mathrm{ES}}{ }^{i}\right) \mathcal{P}_{\mathrm{SCF}}\left(\mathrm{NN}_{\text {out }}{ }^{i}\right),
\end{array}
$$

where $f_{\mathrm{SCF}}$ is the SCF fraction. The extended likelihood function is

$$
\mathcal{L}=\prod_{k} e^{-n_{k}} \prod_{i}\left[\sum_{j} n_{j} \mathcal{P}_{j}^{i}\right],
$$

where $n_{j(k)}$ is the yield of the event category $j(k)$.

For the signal, the $m_{\mathrm{ES}}$ PDFs for CR and SCF are described by an asymmetric Gaussian with power-law tails and a third-order Chebyshev polynomial, respectively. Both CR and SCF $\mathrm{NN}_{\text {out }}$ PDFs are described by nonparametric PDFs (one-dimensional histograms). We fix the shape parameters of the signal $m_{\mathrm{ES}}$ PDFs to the values obtained from the $B^{+} \rightarrow K^{+} \pi^{0} \pi^{0}$ phase-space MC sample. The parameters are corrected to account for possible differences between data and MC simulations, using correction factors determined with a high-statistics control sample of $B^{+} \rightarrow \bar{D}^{0} \rho^{+} \rightarrow\left(K^{+} \pi^{-} \pi^{0}\right)\left(\pi^{+} \pi^{0}\right)$ decays. For the continuum background, we use an ARGUS function [32] to parameterize the $m_{\mathrm{ES}}$ shape. The endpoint of the ARGUS function is fixed to $5.289 \mathrm{GeV} / c^{2}$ whereas the shape parameter is allowed to float in the fit. The continuum $\mathrm{NN}_{\text {out }}$ shape is modelled with a 20 bin parametric step function, i.e., a histogram with non-uniform bin width and variable bin content. Onedimensional histograms are used as nonparametric PDFs to represent all fit variables for the four $B \bar{B}$ background components. The free parameters of our fit are the yields of signal and continuum background together with the parameters of the continuum $m_{\mathrm{ES}}$ and $\mathrm{NN}_{\text {out }}$ PDFs. All yields and PDF shapes of the four $B \bar{B}$ background categories are fixed to values based on MC simulations.

The results of the fit are highly sensitive to the value of $f_{\mathrm{SCF}}$, which depends strongly on the Dalitz plot distribution of signal events and cannot be determined directly from the fit. To circumvent this problem, we adopt an iterative procedure. We perform a fit with $f_{\mathrm{SCF}}$ fixed to an initial value. We then construct the signal Dalitz plot from the signal probabilities for each candidate event $\left({ }_{s} \mathcal{W}\right.$ eights $)$ calculated with the ${ }_{s} \mathcal{P}$ lot technique [33], and determine the corresponding average value of $f_{\mathrm{SCF}}$. We then fit again with $f_{\mathrm{SCF}}$ fixed to the new value, and repeat until the obtained values of the total signal yield $(\mathrm{CR}+\mathrm{SCF})$ and $f_{\mathrm{SCF}}$ are unchanged between iterations. This method was validated using $\mathrm{MC}$ and was found to return values of $f_{\mathrm{SCF}}$ that are accurate to within $3 \%$ of the nominal SCF fraction. Convergence is typically obtained within three iterations.

We cross-check our analysis procedure using the high statistics control sample described above. We impose selection requirements on the $D$ and $\rho$ candidates' invariant masses: $1.84 \mathrm{GeV} / c^{2}<m_{K^{+} \pi^{-} \pi^{0}}<1.88 \mathrm{GeV} / c^{2}$ and $0.65 \mathrm{GeV} / c^{2}<m_{\pi^{+} \pi^{0}}<0.85 \mathrm{GeV} / c^{2}$. We fit the on-peak data with a likelihood function that includes components for the control sample, all $B \bar{B}$ backgrounds, and $q \bar{q}$. We find a yield that is consistent with expectation based on the world-average branching fractions [29].

We apply the fit method described above to the 31673 selected candidate $B^{+} \rightarrow K^{+} \pi^{0} \pi^{0}$ events. Convergence is obtained after four iterations with a yield of $1220 \pm 85$ signal events and a SCF fraction of $9.7 \%$. The results of the fit are shown in Fig. 2. The statistical significance of the signal yield, given by $\sqrt{2 \Delta \ln \mathcal{L}}$ where $\Delta \ln \mathcal{L}$ is the difference between the negative log likelihood obtained assuming zero signal events and that at its minimum, is 15.6 standard deviations $(\sigma)$. Including systematic uncertainties (discussed below), the significance is above $10 \sigma$.

To obtain the $B^{+} \rightarrow K^{+} \pi^{0} \pi^{0}$ branching fraction using the result of the fit, we form, for each event, the ratio of the signal ${ }_{s} \mathcal{W}$ eight and the efficiency determined from its Dalitz-plot position. Summing these ratios over all events in the data sample, we obtain an efficiencycorrected signal yield of $7427 \pm 518$ events. The ${ }_{s} \mathcal{W}$ eight calculation accounts for the fixed $B \bar{B}$ backgrounds [33]. The Dalitz plot distributions obtained before and after applying the efficiency correction are shown in Fig. 3. We apply further corrections for the effect of the $K_{S}^{0}$ veto (98\%); differences between data and MC for the $\pi^{0}$ reconstruction efficiency, determined from control samples of $\tau$ decays as a function of $\pi^{0}$ momentum (95.7\%); and a bias in the fitted signal yield (raw bias 44 events), as determined from Monte Carlo pseudoexperiments generated with a signal component with the same values of the yield and SCF fraction as found in the fit to data. Finally, we divide by the total number of $B \bar{B}$ events in the data sample to obtain our measurement of the branching fraction $\mathcal{B}\left(B^{+} \rightarrow K^{+} \pi^{0} \pi^{0}\right)=(16.2 \pm 1.2 \pm 1.5) \times 10^{-6}$, where the first uncertainty is statistical and the second is systematic.

The systematic uncertainty includes contributions from the PDF shapes; the fixed $B \bar{B}$ background yields; the estimation of the SCF fraction; intrinsic fit bias; selection requirements; and the number of $B \bar{B}$ pairs in the data sample. Here we provide further details on each of these sources of systematic uncertainty and describe briefly how each is evaluated. A combined uncertainty for CR signal and $B \bar{B}$ background $\mathrm{NN}_{\text {out }} \mathrm{PDF}$ shapes $(4.9 \%)$ is evaluated using uncertainties in the data/MC ratio determined from the $B^{+} \rightarrow \bar{D}^{0} \rho^{+} \rightarrow$ $\left(K^{+} \pi^{-} \pi^{0}\right)\left(\pi^{+} \pi^{0}\right)$ control sample and applying them simultaneously to the $\mathrm{CR}$ signal and $B \bar{B}$ background $\mathrm{NN}_{\text {out }}$ PDFs. The same control sample is used to evaluate the uncertainties in $\mathrm{CR}$ signal $m_{\mathrm{ES}} \mathrm{PDF}$ shapes 

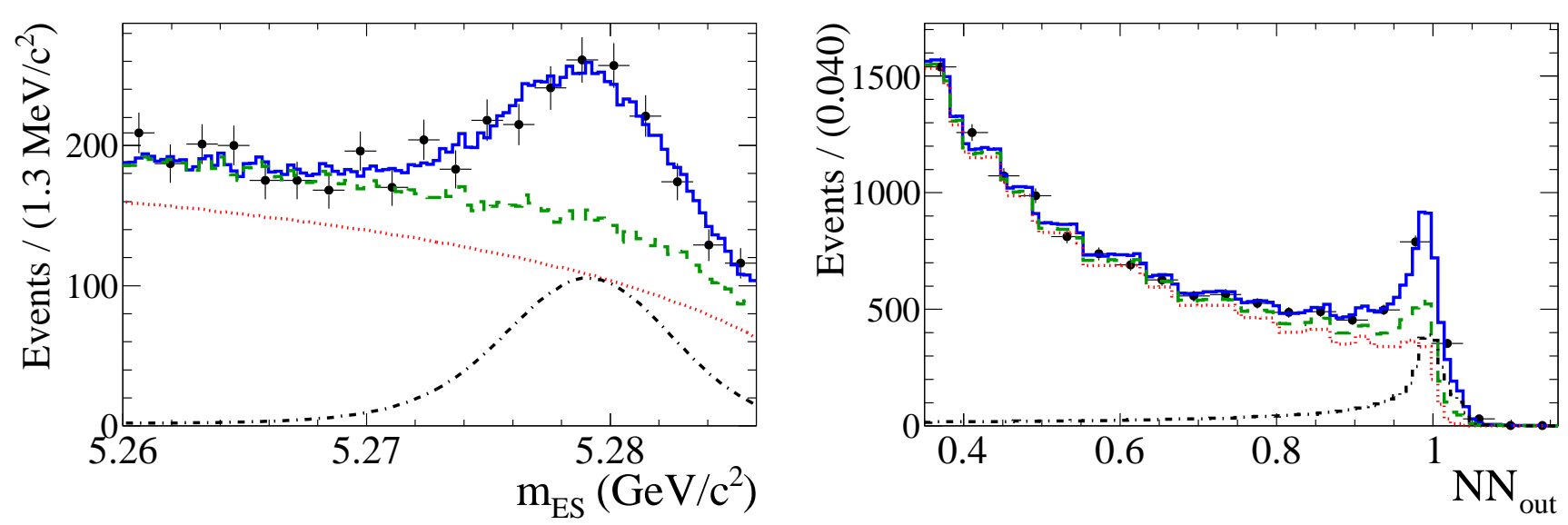

FIG. 2: Projections of candidate events onto $m_{\mathrm{ES}}$ (left) and $\mathrm{NN}_{\text {out }}$ (right), following requirements on the other fit variable that enhance signal visibility. These requirements retain $60 \%$ of signal events for the $m_{\mathrm{ES}}$ plot and $87 \%$ of events for the $\mathrm{NN}_{\mathrm{out}}$ plot. Points with error bars show the data, the solid (blue) lines the total fit result, the dashed (green) lines the total background contribution, and the dotted (red) lines the $q \bar{q}$ component. The dash-dotted lines represent the signal contribution.
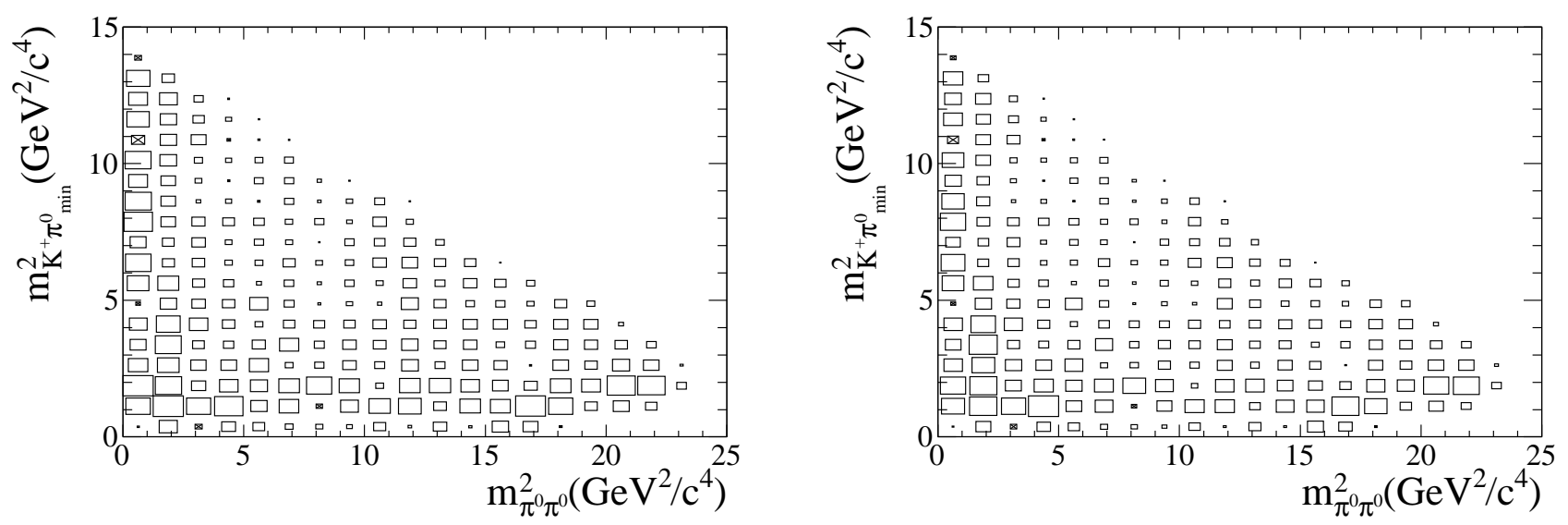

FIG. 3: Signal Dalitz plot distributions obtained using ${ }_{s} \mathcal{W}$ eights before (left) and after (right) efficiency correction. In order to define a unique position for each event, the Dalitz plot is shown as $m_{K^{+} \pi_{\min }^{0}}^{2}$ vs. $m_{\pi^{0} \pi^{0}}^{2}$, where $m_{K^{+} \pi_{\min }^{0}}^{2}$ is the smaller of the two $K^{+} \pi^{0}$ invariant masses. Resonance bands are visible for $K^{*}(892)^{+}$at $m_{K^{+} \pi_{\min }^{0}}^{2} \sim 0.8 \mathrm{GeV}^{2} / \mathrm{c}^{4}, f_{0}(980)$ at $m_{\pi^{0} \pi^{0}}^{2} \sim 1 \mathrm{GeV}^{2} / \mathrm{c}^{4}$ and $\chi_{c 0}$ at $m_{\pi^{0} \pi^{0}}^{2} \sim 12 \mathrm{GeV}^{2} / \mathrm{c}^{4}$.

$(0.8 \%)$. The uncertainty in the SCF fraction $(2.5 \%)$ is estimated by varying the value used in the fit within a range of uncertainty determined from Monte Carlo pseudoexperiment tests of our iterative fitting procedure. Uncertainties in the SCF signal $m_{\mathrm{ES}}$ and $\mathrm{NN}_{\text {out }}$ PDF shapes ( $1.7 \%$ and $0.7 \%$, respectively) are evaluated by considering a range of SCF shapes corresponding to different signal Dalitz plot distributions. An uncertainty in the correction due to fit bias $(1.9 \%)$ is assigned, which corresponds to half the correction combined in quadrature with its error. Uncertainties in the $B \bar{B}$ background $m_{\mathrm{ES}}$ PDF shapes due to data/MC differences (1.6\%) are evaluated by smearing the PDFs with a Gaussian with parameters determined from the $B^{+} \rightarrow \bar{D}^{0} \rho^{+}$control sample. The uncertainties in the $B \bar{B}$ background PDFs due to finite $\mathrm{MC}$ statistics $(0.8 \%)$ are determined by varying the contents of the bins of the histograms used to describe the PDFs within their errors. Uncertainties in the fixed $B \bar{B}$ background yields (1.4\%) are evaluated by varying these yields within their uncertainties. Contributions to the uncertainty in the selection efficiency arise from the $\Delta E(4.0 \%)$ and $\mathrm{NN}_{\text {out }}(3.0 \%)$ selection requirements, neutral pion reconstruction $(2.8 \%)$, the $K_{S}^{0}$ veto correction $(2.0 \%)$, kaon identification $(1.0 \%)$ and tracking $(0.4 \%)$; The uncertainty in the number of $B \bar{B}$ pairs in the data sample is $0.6 \%$. Including only systematic uncertainties that affect the fitted yield, the total is $6.5 \%$. The total systematic uncertainty on the branching fraction is $9.0 \%$. Table II summarizes the systematic contributions. 
TABLE II: Summary of systematic uncertainties for the inclusive branching fraction measurement.

\begin{tabular}{l|c}
\hline Source & Uncertainty \\
\hline CR signal and $B \bar{B}$ background $\mathrm{NN}_{\text {out }}$ PDFs & $4.9 \%$ \\
CR signal $m_{\mathrm{ES}} \mathrm{PDF}$ & $0.8 \%$ \\
$\mathrm{SCF}$ fraction & $2.5 \%$ \\
$\mathrm{SCF}$ signal $m_{\mathrm{ES}} \mathrm{PDF}$ & $1.7 \%$ \\
$\mathrm{SCF}$ signal NN $\mathrm{out}$ PDF & $0.7 \%$ \\
Fit bias & $1.9 \%$ \\
$B \bar{B}$ background $m_{\mathrm{ES}}$ PDFs & $1.6 \%$ \\
$B \bar{B}$ background PDFs (MC statistics) & $0.8 \%$ \\
$B \bar{B}$ background yields & $1.4 \%$ \\
\hline Subtotal & $6.5 \%$ \\
\hline$\Delta E$ selection efficiency & $4.0 \%$ \\
$\mathrm{NN}$ out selection efficiency & $3.0 \%$ \\
Neutral pion efficiency & $2.8 \%$ \\
$K_{S}^{0}$ veto & $2.0 \%$ \\
Particle identification efficiency & $1.0 \%$ \\
Tracking efficiency & $0.4 \%$ \\
$N_{B} \bar{B}$ & $0.6 \%$ \\
\hline Total & $9.0 \%$ \\
\hline
\end{tabular}

The $C P$ asymmetry is measured as

$$
A_{C P}=\frac{N_{B^{-}}-N_{B^{+}}}{N_{B^{-}}+N_{B^{+}}},
$$

where $N_{B^{+}\left(B^{-}\right)}$is the number of events from $B^{+} \rightarrow K^{+} \pi^{0} \pi^{0}$ (CP conjugate decay) and is obtained by including in the above-described fit the value of the kaon charge. The fit returns an asymmetry of $A_{C P}=$ $-0.06 \pm 0.06 \pm 0.04$. Most of the systematic uncertainties that affect the branching fraction cancel in the asymmetry. However, the following sources are considered and evaluated for the $A_{C P}$ measurement. Detector-induced asymmetries have been studied in previous similar analyses $[15,16]$ and found to be small $(0.5 \%)$. We evaluate the possibility that our selection induces an asymmetry by measuring the $C P$ asymmetry in the $B^{+} \rightarrow \bar{D}^{0} \rho^{+}$ control sample $(3.0 \%)$, where none is expected. The $B \bar{B}$ background asymmetries are fixed in our fit; the uncertainty from this is evaluated $(1.8 \%)$ by varying these by a weighted average of the $C P$ asymmetries of the contributing $B \bar{B}$ decays. Finally the fit bias is estimated from $\mathrm{MC}$ pseudoexperiments (1.2\%).

\section{STUDY OF QUASI-TWO-BODY CONTRIBUTIONS}

We use the ${ }_{s} \mathcal{P}$ lot distributions obtained from the fit and projected onto the Dalitz plot axes to search for peaks from intermediate resonances. These projections are shown for both $K^{+} \pi^{0}$ and $\pi^{0} \pi^{0}$ invariant masses in Fig. 4. Signal peaks from $K^{*}(892)^{+}, f_{0}(980)$ and $\chi_{c 0}$ are clearly observed. We do not see any enhancement that could be attributed to the $f_{\mathrm{X}}(1300)$, though the $\pi^{0} \pi^{0}$ invariant mass distribution contains a pronounced dip around $1550 \mathrm{MeV} / \mathrm{c}^{2}$ that could arise from interference between various resonances in this region. A broad peak around $1400 \mathrm{MeV} / c^{2}$ in the $K^{+} \pi^{0}$ invariant mass distribution could be due to contributions from spin-0 and/or spin- $2 K^{*}(1430)^{+}$states.

The numbers of signal events for the quasi-two-body contributions are determined by defining signal regions around the peaks of the resonances. Efficiency-corrected ${ }_{s} \mathcal{W}$ eights are summed in the same way as used to measure the inclusive branching fraction. To estimate contributions from nonresonant and resonant $B^{+} \rightarrow K^{+} \pi^{0} \pi^{0}$ decays other than the quasi-two-body decays under consideration (which we refer to as background in this section), the same procedure is applied to sidebands on either side of each signal region in the two-particle invariant mass. The background yields are estimated as the normalized averages of the two sidebands' yields and are subtracted from the efficiency-corrected yields in the signal regions. The signal and sideband regions are illustrated by arrows for each of the three quasi-two-body modes in Fig. 5. We use this approach rather than a full Dalitz plot analysis since the latter would require more detailed understanding of the properties of SCF events. Our method does, however, suffer from systematic uncertainties (evaluated below) due to other contributions to the Dalitz plot and possible interference effects. This precludes its use for studying quasi-two-body decays via broad resonances. We have validated our approach using ensembles of MC simulations with varying mixtures of resonant substructure, and find that in all cases we are able to correctly obtain the true values of the branching fractions of the quasi-two-body decays under study, which all have narrow intermediate states under study.

Fits to the efficiency-corrected invariant mass distributions are used to cross-check the results of the subtraction method. In these fits we describe the signal distributions with double-Gaussian functions, with parameters obtained from MC simulations, and the background shapes with polynomials. The two methods yield consistent results, both in MC simulations and in data.

After background subtraction we obtain efficiency-corrected signal yields of $1078 \pm 197$ for $B^{+} \rightarrow K^{*}(892)^{+} \pi^{0}, 1186 \pm 241$ for $B^{+} \rightarrow f_{0}(980) K^{+}$, and $245 \pm 105$ for $B^{+} \rightarrow \chi_{c 0} K^{+}$. We correct each yield for the inefficiency of the corresponding signal region selection, obtained from Monte Carlo simulations. Finally the yields are corrected: (i) for bias, estimated from Monte Carlo pseudoexperiments; (ii) for $\pi^{0}$ efficiency, using the momentum distributions of both $\pi^{0}$ mesons from a Monte Carlo cocktail reflecting the yields obtained in data; (iii) in the case of the $K^{*}(892)^{+}$yield only, for the $K_{S}^{0}$ veto. Finally, we divide by the number 

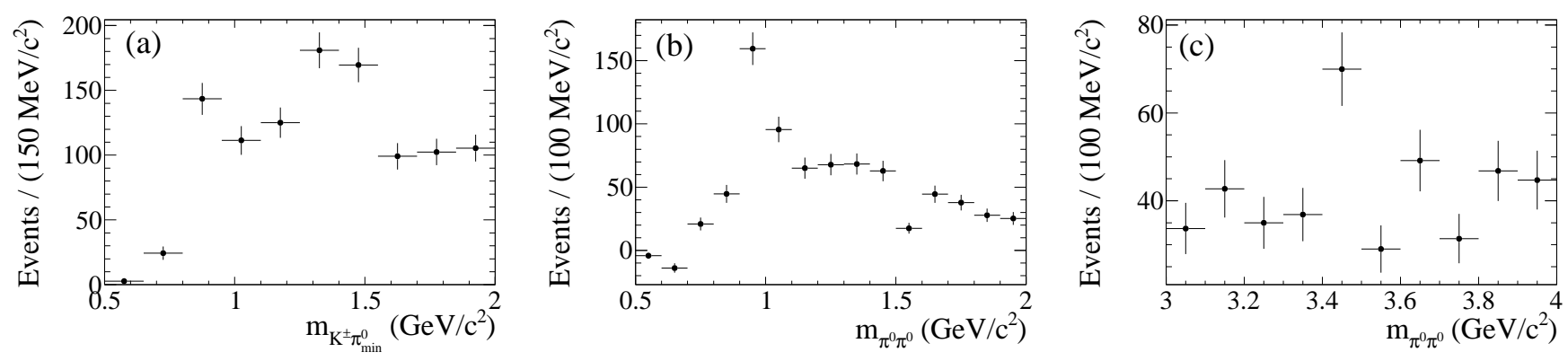

FIG. 4: Signal ${ }_{s} \mathcal{P}$ lot distributions not corrected for efficiency for (a) $0.5 \mathrm{GeV} / c^{2}<m_{K^{+} \pi_{\min }^{0}}<2.0 \mathrm{GeV} / c^{2}$, (b) $0.5 \mathrm{GeV} / c^{2}<$ $m_{\pi^{0} \pi^{0}}<2.0 \mathrm{GeV} / c^{2}$ and (c) $3.0 \mathrm{GeV} / c^{2}<m_{\pi^{0} \pi^{0}}<4.0 \mathrm{GeV} / c^{2} \cdot m_{K^{+} \pi^{0} \text { min }}$ is the $K^{+} \pi^{0}$ combination with lower invariant mass. Excesses of events in the $f_{0}(980), \chi_{c 0}, K^{*}(892)^{+}$, and $K^{*}(1430)^{+}$mass regions are clearly visible.

of $B \bar{B}$ pairs to obtain the product branching fractions

$$
\begin{gathered}
\mathcal{B}\left(B^{+} \rightarrow K^{*}(892)^{+} \pi^{0}\right) \times \mathcal{B}\left(K^{*}(892)^{+} \rightarrow K^{+} \pi^{0}\right)= \\
(2.7 \pm 0.5 \pm 0.4) \times 10^{-6} \\
\mathcal{B}\left(B^{+} \rightarrow f_{0}(980) K^{+}\right) \times \mathcal{B}\left(f_{0}(980) \rightarrow \pi^{0} \pi^{0}\right)= \\
(2.8 \pm 0.6 \pm 0.5) \times 10^{-6} \\
\mathcal{B}\left(B^{+} \rightarrow \chi_{c 0} K^{+}\right) \times \mathcal{B}\left(\chi_{c 0} \rightarrow \pi^{0} \pi^{0}\right)= \\
(0.51 \pm 0.22 \pm 0.09) \times 10^{-6}
\end{gathered}
$$

where the first uncertainties are statistical and the second systematic. The sum of these contributions does not saturate the inclusive branching fraction, indicating significant contributions from other sources, as is also clear from Fig. 3 and Fig. 4, and expected from the results of studies of $B^{+} \rightarrow K^{+} \pi^{+} \pi^{-}$decays $[16,17]$.

Systematic uncertainties include all the same sources in the same relative amounts as evaluated for the inclusive decay except for fit bias, $K_{S}^{0}$ veto and $\pi^{0}$ efficiency, which are evaluated separately for each quasi-two-body mode. We also evaluate the following additional contributions. The uncertainty due to the method of background subtraction $\left(3.5 \%\right.$ for $K^{*}(892)^{+} \pi^{0}, 11.9 \%$ for $f_{0}(980) K^{+}$and $13.5 \%$ for $\left.\chi_{c 0} K^{+}\right)$is obtained by comparing the nominal results with those from obtained with alternative sideband regions. We evaluate the potential effect of interference $\left(10.0 \%\right.$, for $f_{0}(980) K^{+}$only) using toy Monte Carlo events generated for a Dalitz plot model containing $f_{0}(980)$ and nonresonant components with relative magnitudes obtained from the fit results, and a relative phase sampled in a range that gives distributions consistent with the data. Finally we consider possible data/MC differences affecting the signal region efficiency correction $\left(5.6 \%\right.$ for $K^{*}(892)^{+} \pi^{0}, 3.8 \%$ for $f_{0}(980) K^{+}$, and $0.4 \%$ for $\chi_{c 0} K^{+}$) determined from the change in the result when the SCF fraction is varied in Monte Carlo events. The $K^{*}(892)^{+} \pi^{0}$ and $\chi_{c 0} K^{+}$branching fraction measurements are not affected by systematics due to interference. For the former, effects of interference with $K^{+} \pi^{0}$ S-wave contributions cancel when integrated over the part of the Dalitz plot inside the signal mass window, while $\mathrm{P}$-wave contribution are not expected based on studies of related decays $[16,17]$. For the latter, the small width implies that interference will be negligible.
TABLE III: Summary of systematic uncertainties for the branching fraction measurement of the quasi-two-body resonances. The breakdown of the systematics affecting the inclusive branching fraction measurement is given in Table II.

\begin{tabular}{l|ccc}
\hline Source & \multicolumn{3}{|c}{ Uncertainty (\%) } \\
& $K^{*}(892)^{+} \pi^{0}$ & $f_{0}(980) K^{+}$ & $\chi_{c 0} K^{+}$ \\
\hline Subtotal from inclusive & 8.1 & 8.1 & 8.1 \\
\hline Background subtraction & 3.5 & 11.9 & 13.5 \\
Interference & - & 10.0 & - \\
Fit bias & 6.6 & 2.1 & 6.8 \\
Mass cut efficiency & 5.6 & 3.8 & 0.4 \\
$\pi^{0}$ efficiency & 3.1 & 3.5 & 2.6 \\
$K_{S}^{0}$ veto & 2.0 & - & - \\
\hline Total & 12.9 & 18.4 & 17.4 \\
\hline
\end{tabular}

A list of the systematic uncertainty contributions is given in Table III.

To obtain the $B$ decay branching fractions, we correct for $\mathcal{B}\left(K^{*}(892)^{+} \rightarrow K^{+} \pi^{0}\right)=1 / 3$ and $\mathcal{B}\left(\chi_{c 0} \rightarrow \pi^{0} \pi^{0}\right)=$ $(8.4 \pm 0.4) \times 10^{-3} \times 1 / 3[29]$, where the factors of $1 / 3$ are due to isospin. (The branching fraction of $f_{0}(980) \rightarrow$ $\pi^{0} \pi^{0}$ is unknown, hence we cannot correct for it.) We obtain

$$
\begin{aligned}
\mathcal{B}\left(B^{+} \rightarrow K^{*}(892)^{+} \pi^{0}\right) & =(8.2 \pm 1.5 \pm 1.1) \times 10^{-6}, \\
\mathcal{B}\left(B^{+} \rightarrow \chi_{c 0} K^{+}\right) & =(18 \pm 8 \pm 3 \pm 1) \times 10^{-5},
\end{aligned}
$$

where the first uncertainty is statistical, the second systematic, and the third (for $B^{+} \rightarrow \chi_{c 0} K^{+}$) is from the subdecay branching fraction.

We obtain the $C P$ asymmetries of the quasi-two-body modes with the same method used to obtain the quasitwo-body branching fractions, except we distinguish the yields of the $B^{+}$and $B^{-}$decays. We obtain the following asymmetries:

$$
\begin{gathered}
A_{C P}\left(B^{+} \rightarrow K^{*}(892)^{+} \pi^{0}\right)=-0.06 \pm 0.24 \pm 0.04 \\
A_{C P}\left(B^{+} \rightarrow f_{0}(980) K^{+}\right)=0.18 \pm 0.18 \pm 0.04 \\
A_{C P}\left(B^{+} \rightarrow \chi_{c 0} K^{+}\right)=-0.96 \pm 0.37 \pm 0.04
\end{gathered}
$$

where the first uncertainty is statistical and the second systematic. The sources of systematic uncertainty 

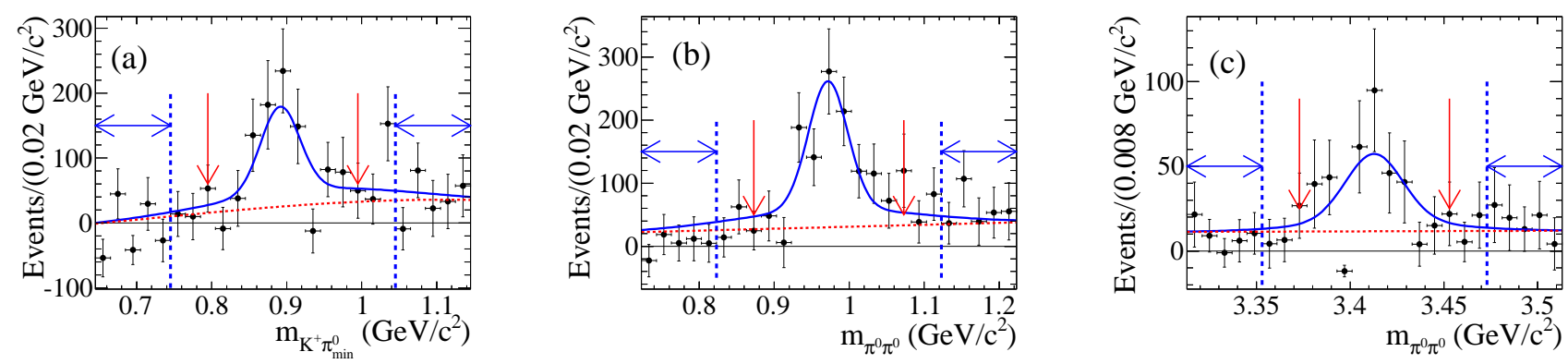

FIG. 5: Efficiency-corrected signal (vertical red arrows) and sideband (horizontal blue arrows) regions around (a) $K^{*}(892)^{+}$, (b) $f_{0}(980)$ and (c) $\chi_{c 0}$ invariant mass peaks. The curves show the results of the fit used to cross-check the procedure, for the total (blue continuous line) and background-only (dashed red line) components.

are the same as for the inclusive $C P$ asymmetry measurement. The measurements of $C P$ asymmetries for $B^{+} \rightarrow f_{0}(980) K^{+}$and $B^{+} \rightarrow \chi_{c 0} K^{+}$are consistent with the world average values based on decays of the intermediate resonances to $\pi^{+} \pi^{-}[10,29]$. The $B^{+} \rightarrow \chi_{c 0} K^{+}$ result has a large and non-Gaussian uncertainty and its difference from zero is not statistically significant.

\section{CONCLUSION}

In summary, using the full BABAR data sample of $429 \mathrm{fb}^{-1}$ collected at the $\Upsilon(4 S)$ resonance, we observe charmless hadronic decays of charged $B$ mesons to the final state $K^{+} \pi^{0} \pi^{0}$. The signal has a significance above $10 \sigma$ after taking systematic effects into account.

We study the Dalitz plot distribution of the signal events, and do not see any excess that could be attributed to the $f_{\mathrm{X}}(1300)$. However, due to the possible complicated interference pattern, we cannot draw any strong conclusion about this state from our analysis. We measure the product branching fractions and direct $C P$ asymmetry parameters of the quasi-two-body modes with narrow resonance peaks in the $K^{+} \pi^{0} \pi^{0}$ Dalitz plot.

The results are summarized in Table IV. All measured $C P$ asymmetries are consistent with zero. The branching fraction result for $B^{+} \rightarrow \chi_{c 0} K^{+}$is consistent with the world average, while that for $B^{+} \rightarrow K^{*}(892)^{+} \pi^{0}$ is consistent with and more precise than our previous measurement [15], which it supersedes. 
TABLE IV: Summary of measurements of branching fractions (averaged over charge conjugate states) and $C P$ asymmetries. Both product branching fractions and those corrected for secondary decays are shown. For each result, the first uncertainty is statistical, the second is systematic and the third, where quoted, is the error on $\chi_{c 0} \rightarrow \pi^{0} \pi^{0}$. The notation $R h$ refers, where applicable, to the intermediate state of a resonance and a bachelor hadron.

\begin{tabular}{lccr}
\hline Mode & $\mathcal{B}\left(B^{+} \rightarrow R h \rightarrow K^{+} \pi^{0} \pi^{0}\right)$ & $\mathcal{B}\left(B^{+} \rightarrow R h\right)$ & \multicolumn{1}{c}{$A_{C P}$} \\
\hline$B^{+} \rightarrow K^{+} \pi^{0} \pi^{0}$ & $(16.2 \pm 1.2 \pm 1.5) \times 10^{-6}$ & $\cdots$ & $-0.06 \pm 0.06 \pm 0.04$ \\
\hline$B^{+} \rightarrow K^{*}(892)^{+} \pi^{0}$ & $(2.7 \pm 0.5 \pm 0.4) \times 10^{-6}$ & $(8.2 \pm 1.5 \pm 1.1) \times 10^{-6}$ & $-0.06 \pm 0.24 \pm 0.04$ \\
$B^{+} \rightarrow f_{0}(980) K^{+}$ & $(2.8 \pm 0.6 \pm 0.5) \times 10^{-6}$ & $\ldots$ & $0.18 \pm 0.18 \pm 0.04$ \\
$B^{+} \rightarrow \chi_{c 0} K^{+}$ & $(0.51 \pm 0.22 \pm 0.09) \times 10^{-6}$ & $(18 \pm 8 \pm 3 \pm 1) \times 10^{-5}$ & $-0.96 \pm 0.37 \pm 0.04$ \\
\hline
\end{tabular}


We are grateful for the extraordinary contributions of our PEP-II colleagues in achieving the excellent luminosity and machine conditions that have made this work possible. The success of this project also relies critically on the expertise and dedication of the computing organizations that support BABAR. The collaborating institutions wish to thank SLAC for its support and the kind hospitality extended to them. This work is supported by the US Department of Energy and National Science Foundation, the Natural Sciences and Engineering Research Council (Canada), the Commissariat à l'Energie Atomique and Institut National de Physique Nucléaire et de Physique des Particules (France), the Bundesministerium für Bildung und Forschung and Deutsche Forschungsgemeinschaft (Germany), the Istituto Nazionale di Fisica Nucleare (Italy), the Foundation for Fundamental Research on Matter (The Netherlands), the Research Council of Norway, the Ministry of Education and Science of the Russian Federation, Ministerio de Ciencia e Innovación (Spain), and the Science and Technology Facilities Council (United Kingdom). Individuals have received support from the Marie-Curie IEF program (European Union), the A. P. Sloan Foundation (USA) and the Binational Science Foundation (USA-Israel).
[1] B. Aubert et al. (BABAR Collaboration), Phys. Rev. D76, 091102 (2007), arXiv:0707.2798 [hep-ex].

[2] S. W. Lin et al. (Belle Collaboration), Nature 452, 332 (2008).

[3] R. Fleischer, S. Jager, D. Pirjol, and J. Zupan, Phys. Rev. D78, 111501 (2008), arXiv:0806.2900 [hep-ph].

[4] M. Gronau and J. L. Rosner, Phys. Lett. B666, 467 (2008), arXiv:0807.3080 [hep-ph].

[5] M. Ciuchini, E. Franco, G. Martinelli, M. Pierini, and L. Silvestrini, Phys. Lett. B674, 197 (2009), arXiv:0811.0341 [hep-ph].

[6] Q. Chang, X.-Q. Li, and Y.-D. Yang, JHEP 09, 038 (2008), arXiv:0807.4295 [hep-ph].

[7] C.-W. Chiang and D. London, Mod. Phys. Lett. A24, 1983 (2009), arXiv:0904.2235 [hep-ph].

[8] M. Gronau, D. Pirjol, and J. Zupan, Phys. Rev. D81, 094011 (2010), arXiv:1001.0702 [hep-ph].

[9] The inclusion of charge conjugate modes is implied throughout this paper.

[10] D. Asner et al. (Heavy Flavor Averaging Group) (2010), arXiv:1010.1589 [hep-ex], URL http://www . slac.stanford.edu/xorg/hfag/.

[11] B. Aubert et al. (BABAR Collaboration), Phys. Rev. D78, 052005 (2008), arXiv:0711.4417 [hep-ex].

[12] B. Aubert et al. (BABAR Collaboration) (2008), arXiv:0807.4567 [hep-ex].

[13] A. Garmash et al. (Belle Collaboration), Phys. Rev. D75, 012006 (2007), hep-ex/0610081.

[14] J. Dalseno et al. (Belle Collaboration), Phys. Rev. D79, 072004 (2009), arXiv:0811.3665 [hep-ex].

[15] B. Aubert et al. (BABAR Collaboration), Phys. Rev. D71, 111101 (2005), hep-ex/0504009.

[16] B. Aubert et al. (BABAR Collaboration), Phys. Rev. D78,
012004 (2008), arXiv:0803.4451 [hep-ex].

[17] A. Garmash et al. (Belle Collaboration), Phys. Rev. Lett. 96, 251803 (2006), hep-ex/0512066.

[18] P. Chang et al. (Belle Collaboration), Phys. Lett. B599, 148 (2004), hep-ex/0406075.

[19] B. Aubert et al. (BABAR Collaboration), Phys. Rev. D80, 112001 (2009), arXiv:0905.3615 [hep-ex].

[20] A. P. Wagner, Ph.D. thesis (2010), SLAC-R-942.

[21] T. Gershon and M. Hazumi, Phys. Lett. B596, 163 (2004), hep-ph/0402097.

[22] B. Aubert et al. (BABAR Collaboration), Phys. Rev. D76, 071101 (2007), hep-ex/0702010.

[23] N. Cabibbo, Phys. Rev. Lett. 10, 531 (1963).

[24] M. Kobayashi and T. Maskawa, Prog. Theor. Phys. 49, 652 (1973).

[25] B. Aubert et al. (BABAR Collaboration), Phys. Rev. D74, 032003 (2006), hep-ex/0605003.

[26] A. Garmash et al. (Belle Collaboration), Phys. Rev. D71, 092003 (2005), hep-ex/0412066.

[27] B. Aubert et al. (BABAR Collaboration), Nucl. Instrum. Methods Phys. Res., Sect. A 479, 1 (2002).

[28] A. Drescher et al., Nucl. Instrum. Meth. A237, 464 (1985).

[29] K. Nakamura et al. (Particle Data Group), J. Phys. G37, 075021 (2010).

[30] B. Aubert et al. (BABAR Collaboration), Phys. Rev. D79, 072009 (2009), arXiv:0902.1708 [hep-ex].

[31] G. Punzi (2004), physics/0401045.

[32] H. Albrecht et al. (ARGUS Collaboration), Phys. Lett. B241, 278 (1990).

[33] M. Pivk and F. R. Le Diberder, Nucl. Instrum. Meth. A555, 356 (2005), physics/0402083. 